\title{
When did everyone become so naughty?
}

\section{With new calls for editors to police author misconduct and declare editorial conflicts, the JCI outlines its current policies and reflects on a few particu- larly salient examples of bad behavior.}

The London-based Committee on Publication Ethics (COPE), established in 1997 to guide editors through ethical issues, recently published a draft of a code for editorial accountability (1). It seems reasonable that there would be a group prepared to help editors deal with misbehaving authors, but apparently authors aren't the only ones behaving badly these days.

The 2003 COPE Report outlines a number of cases in which editors of research journals have been less than honest. One infamous editor wrote letters (later published) under a pseudonym in order to criticize a colleague. One editor reversed another editor's decision to reject a manuscript because of a personal financial conflict of interest. Another editor pushed a biased view of a particular field through publication of 14 review articles by the same author. A section of the report details a year's worth of allegations of scientific fraud reported by the nearly 200 editors participating in COPE.

In light of these reports, we thought we should reiterate our policies on how we handle editorial conflicts and reports of fraudulent behavior. For those of you unfamiliar with how the Editorial Board works, here is a primer: when a manuscript is submitted to the JCI for review, it first crosses my (the Executive Editor's) desk. Alongside the Editor-in-Chief (Andrew Marks), I assign manuscripts to one of the JCI's 19 Editorial Board members. To prevent any institutional bias, I handle all manuscripts with authors on the Columbia University faculty in a separate system, and often I seek guidance from a member of a previous JCI Editorial Board; the first time any of the $J C I$ Editors will see an institutional colleague's manuscript submitted to the JCI is if it is published. I also have a list of all the current Editors' financial conflicts of interest and handle manuscripts with potential conflicts separately. I myself have no financial conflicts that would influence evaluation of any manuscript. I am not aware of any bias or misconduct on the part of any of the JCI Editors, and I urge any author to e-mail me personally with any concerns. Additionally, the Editorial Board of the JCI moves from university to university every five years, not only to reinvigorate the content but to remove any potential biases of a particular Editorial Board.

On author misconduct, the past year has been replete with examples to flesh out our policies. We take allegations of fraud very seriously and have had a longstanding policy of policing mischievous authors. The COPE Report asks all Editors to investigate and notify authors' superiors when allegations are made. (The JCI does not participate in COPE but agrees with this sentiment.) In view of this, we have made use of the US-based Office of Research Integrity (ORI) (2), within the Department of Health and Human Services. The ORI has clear policies in place for whistleblowers, and it has been invaluable in giving advice to the JCI in how to proceed.

Once we contact the ORI, the office assesses the allegation and recommends a course of action - often a Research Integrity Officer (RIO) at the authors' institution is contacted to assess the claim. The RIO can then launch an inquiry to determine if a formal investigation is required. After evaluating the case, the RIO usually compiles a report and is able to provide us with all the details required to make a decision on whether any action (such as a correction or retraction) is needed.

This past year we received an anonymous letter, written in block capital letters, pointing us to a suspicious figure in a JCI research article. After an RIO investigated, it was determined that while the published blots appeared to be different exposures derived from the same gel, the authors had many replicates of the experiment showing the same pattern of expression. A correction was published. Another case in which authors have been accused of reusing control data previously published in another article is pending. In a more extreme case, authors retracted their JCI paper after an independent committee determined that several of the experiments were not approved by their institutional animal care and usage committee (3).

An interesting test of our ethical policies was encountered when we sent a manuscript for review to a referee who had previously reviewed the same article for a dif- ferent journal. The referee claimed that the original version contained further control data, which invalidated the central hypothesis of the paper. Was the referee correct to point this out? Should the referee have recused himself or herself from the review process? Were the authors acting ethically to selectively exclude this data? Should authors assume that each review they get from a journal will be a fresh perspective? In the end, we asked the authors to support their claims with data - which, when provided, helped to guide our decision.

The most common allegations of misconduct relate to authorship: postdoctoral fellows who have left the labs in which the work was initiated later submit their work to the JCI without the senior author's name (or approval, usually). Investigations into where and when the work was performed, and by whom, can be extensive, and the intellectual property disputes can be difficult to sort out. It goes without saying that "stealing" data can have profound repercussions.

There are, of course, occasions when the authors are accused of mischief but are innocent. Recently a referee noticed that about 50 data points, representing different patients and all registering near-zero values, were removed from a graph during revision. The ORI counseled us to contact the senior author, who was able to provide us with the primary data showing that the original graph had included zeros instead of placeholders: the 50 patients registering zero values had never existed. The authors were very forthcoming and apologetic - we realized that, overall, most people are presenting us with honest, incontrovertible data.

\section{Ushma Savla, Ph.D. Executive Editor}

1. Office of Research Integrity. U.S. Department of Health \& Human Services. http://www. publicationethics.org.uk/cope2003/pages2003/ contents.phtml.

2. Committee on Publication Ethics. The COPE report 2003. http://ori.hhs.gov/.

3. Savla, U. 2003. Responsible conduct in animal research. J. Clin. Invest. 112:1456. doi:10.1172/ JCI200320394. 